\title{
BMJ Open Study protocol for a randomised controlled trial to test the effectiveness of providing information on childbirth and postnatal period to partners of pregnant women
}

\author{
Kenji Takehara, ${ }^{1}$ Makoto Okamura, ${ }^{2}$ Naomi Sugiura, ${ }^{2}$ Maiko Suto, ${ }^{3}$ Hatoko Sasaki, ${ }^{1}$ \\ Rintaro Mori ${ }^{1}$
}

To cite: Takehara $\mathrm{K}$, Okamura M, Sugiura N, et al. Study protocol for a randomised controlled trial to test the effectiveness of providing information on childbirth and postnatal period to partners of pregnant women. BMJ Open 2016;6:e11919.

doi:10.1136/bmjopen-2016011919

- Prepublication history for this paper is available online. To view these files please visit the journal online (http://dx.doi.org/10.1136/ bmjopen-2016-011919).

Received 16 March 2016 Revised 25 May 2016 Accepted 1 July 2016

CrossMark

\footnotetext{
${ }^{1}$ Department of Health Policy, National Center for Child Health and Development, Tokyo, Japan

${ }^{2}$ Okamura Obstetrics and Gynecology Clinic, Aichi, Japan

${ }^{3}$ Graduate School of International and Cultural Studies, Tsuda College,

Tokyo, Japan
}

Correspondence to Dr Kenji Takehara; takehara-k@ncchd.go.jp

\section{ABSTRACT}

Introduction: The objective of this study is to investigate the effect of the distribution of a booklet providing information to fathers during their partners' pregnancies on fathers' and mothers' postpartum mental health and quality of life (QOL), as well as on fathers' childcare participation and living situations.

Methods and analysis: This randomised controlled trial will comprise 554 couples consisting of pregnant women due to give birth at an obstetric institution in Aichi Prefecture, Japan and their partners. Participants will be recruited during prenatal check-ups in the third trimester, and those who provide written consent will be allocated randomly to an intervention and a control group. The pregnant women's partners allocated to the intervention group will be given a booklet written for men containing information on childbirth and postnatal period. Its content will include matters such as what preparations the partner should make before birth and tips for housework and childcare as well as how to prevent unintentional injury to the baby. The control group will not receive any intervention. A baseline survey in the third trimester and follow-up surveys at 1 and 3 months post partum will be carried out using selfadministered questionnaires. The primary outcome is the proportion of new mothers' partners at risk for paternal depression (Edinburgh Postnatal Depression Scale score $\geq 8$ ). Secondary outcomes include the risk of postnatal depression in new mothers, QOL of new mothers and their partners, partners' knowledge of and engagement in housework and childcare, marital relations and parenting stress on the part of new mothers.

Ethics and dissemination: This study has been approved by the Ethical Committee at the National Center for Child Health and Development, Tokyo, Japan. The results of the study will be widely disseminated as peer-reviewed papers and at international conferences, with the aim of improving public health services in Japan.

Trial registration number: UMIN000021475; Pre-results.

\section{Strengths and limitations of this study}

- Adequate measures for preventing paternal depression have yet to be identified, and this study will verify the effectiveness of providing appropriate information to partners during pregnancy.

- Since data will be gathered from couples, the study will verify the effect of this intervention on pregnant women and new mothers as well as their partners.

- Unlike prevention programmes that use methods such as parenting classes or counselling for partners, information in this study is provided in the form of a booklet, making it easy to extend to other institutions.

- This trial will be performed at a single institution, meaning that there are limitations as to the generalisation of the results. Since the intervention programme consists of the distribution of a booklet, there is a high probability of ineffectiveness if the participants fail to read it.

- The staff of the participating institution and the participants cannot be blinded.

\section{INTRODUCTION}

Women's postpartum mental health is an important issue that needs to be addressed. However, it has become evident that both mothers and fathers experience postpartum mental health problems. A meta-analysis of 43 studies involving 28004 fathers published between 1980 and 2009 found that $10.4 \%$ of fathers are judged to be at risk of mental health problems post partum. ${ }^{1}$ In Japan, one study found that $13.6 \%$ of fathers were judged to be at risk of postpartum mental health problems at 4 months after childbirth. ${ }^{2}$ In our previous study, we also found that $16.7 \%$ were at risk of mental health problems at one or more out of five time points between a few days and 3 months post partum. ${ }^{3}$ 
The deterioration of fathers' prenatal and postnatal mental health increases the risk of suicide ${ }^{4}$ and also negatively affects parenting behaviour. ${ }^{5}{ }^{6}$ The possibility has also been raised that fathers' mental health may have various effects on the health of the entire family, including increased risk of adverse effects on the mental health of pregnant women and new mothers ${ }^{78}$ as well as on marital relations, ${ }^{9}$ and negatively affects premature birth, ${ }^{10}$ child development ${ }^{11-13}$ and excessive infant crying. ${ }^{14}$ Conversely, appropriate engagement in childcare by fathers during the postpartum period has been shown to help prevent unintentional injury to children ${ }^{15}$ and enable continued breast feeding. ${ }^{16}$ These factors further indicate the importance of improving fathers' postpartum mental health as a health issue.

Although the development of programmes to prevent maternal depression and their effectiveness has been well described, ${ }^{17}$ the same cannot be said for programmes that combat paternal depression. Randomised controlled trials (RCTs) of prenatal education for partners of pregnant woman have demonstrated the impact on encouragement to stop smoking, ${ }^{18}$ alleviating worries about childbirth, ${ }^{19}$ practice of breast feeding, ${ }^{20}$ alleviation of parenting stress and encouraging engagement in childcare, ${ }^{21}{ }^{22}$ and improvements in marital relations. ${ }^{23}$ However, many of these studies suffered from limitations such as small sample sizes. Two-hour antenatal education sessions and the provision of postnatal social support and information for fathers have been shown to increase the rate of continued breast feeding at 6 weeks post partum ${ }^{24}$ but had no effect in terms of improving fathers' postpartum mental health. ${ }^{25}$ More scientific evidence is thus required on whether providing information to men with pregnant wives is effective.

Although public health services for pregnant women and new mothers, such as prenatal health check-ups, are comparatively well developed in Japan, very few public health services for their male partners are available. However, an increasing number of local governments are issuing 'Father and Child Health Handbook' (Fushi Techo) booklets for men that provide information on pregnancy, childbirth and childcare. The actual effect of the distribution of such booklets to men, however, has yet to be ascertained.

This study will involve a RCT to verify the effect of distributing booklets that provide information on pregnancy, childbirth and childcare to the partners of pregnant women on their mental health and engagement in childcare, mothers' mental health and parenting stress, marital relations and parent-child bonding.

\section{METHODS AND ANALYSIS}

\section{Study design}

This study is a RCT to be carried out at a single obstetric clinic in the suburb of Aichi Prefecture, Japan, where 1080 deliveries were performed in 2015 including 212 deliveries by caesarean section. Participants who provide informed consent will be allocated randomly to an intervention and a control group. Figure 1 shows the flow chart of the study.

\section{Participants and recruitment}

The study participants will consist of pregnant women due to give birth at the above obstetric clinic and their partners, other than those to whom any of the following four exclusion criteria apply: (1) women who underwent their first prenatal health check-up later than 28 weeks gestation; (2) one or both members of the couple are unable to read or write Japanese; (3) individuals undergoing special medical treatment or care for existing mental health problems or poor health due to serious obstetric complications; or (4) those judged by their physician to be unsuitable to participate in the study for other health reasons.

Participant recruitment will be carried out by the staff of the obstetric clinic when the participants visit the clinic for prenatal health check-ups at around 28-32 weeks gestation. The clinical staff will give a simple oral explanation of the study to the participants and hand them an envelope containing an information sheet, consent form and baseline questionnaire. The information sheet illustrates the purpose, procedure and flow chart of the study; it further explains the data management, including linkable

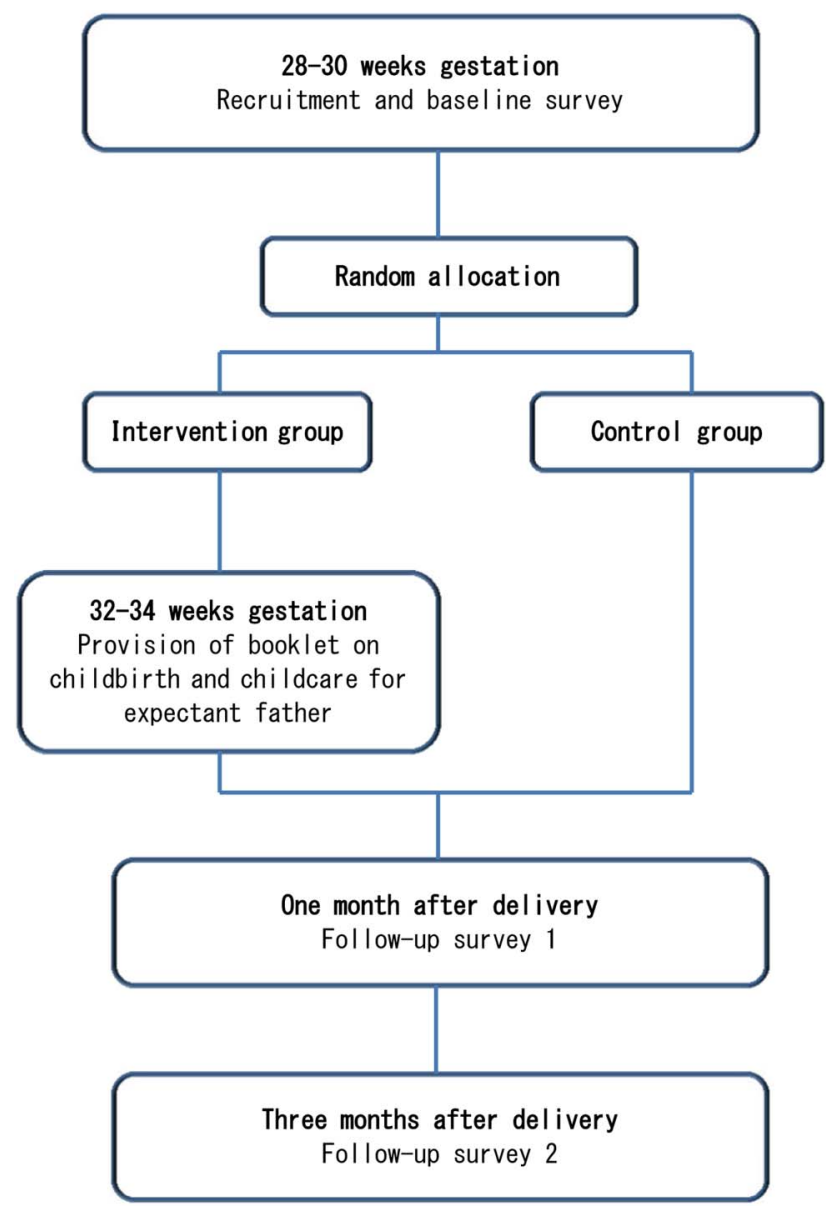

Figure 1 Summary of the study design. 
anonymisation, sharing the data with other facilities including a local governmental agency when their physician considers it necessary to communicate with others to obtain the participant's response from the questionnaires. After being informed about the study, participants will sign the consent form and answer the baseline questionnaire.

\section{Randomisation and blinding}

Randomisation will be carried out by the permuted block method using the RAND function of Microsoft Excel. All the tasks involved in randomisation will be carried out at the National Center for Child Health and Development in Japan, and the staff at the obstetric clinic will have no involvement in the outcome of randomisation. However, it is possible that participants will inform the staff of the results of randomisation when discussing the study with them during visits to the obstetric clinic.

Participants allocated to the intervention group will have a booklet written for men containing information on pregnancy, childbirth and childcare sent to them by post. Since the intervention in this study consists of the distribution of this booklet, the participants cannot be blinded. To prevent contamination, participants in the intervention group will be requested not to talk about the content of the booklet to other couples. To verify the absence of contamination and compliance with allocation, the participants in the intervention and control groups will be asked whether they received the booklet and read it via the questionnaire at the follow-up study 1 and 2.

\section{Data collection}

Data will be collected at three time points: immediately after recruitment at 28-30 weeks gestation and 1 and 3 months after childbirth (follow-up study 1 and follow-up study 2). At these time points, the pregnant women/new mothers and their partners will be asked to respond to a self-administered questionnaire. The completed baseline questionnaire will either be collected by hand by the clinical staff in the same envelope as the consent form or mailed by post to the study institution. The questionnaire for follow-up study 1 will be provided by the clinical staff and collected the same way as the baseline questionnaire. The questionnaire for follow-up study 2 will be sent out and returned by mail. The questionnaires of the follow-up studies will not be provided to mothers who do not give birth and those who do not visit the clinic to receive postnatal check-up at 1 month post partum.

\section{Intervention}

After the baseline survey questionnaires have been collected, the participants in the intervention group will be sent a booklet containing information for fathers on pregnancy, childbirth and childcare by mail from the study institution. This booklet consists of 32 B5 pages including information on topics including (1) the process of childbirth and what preparations are necessary; (2) tips for ensuring smooth communication between husband and wife before and after birth; (3) the process of childbirth when the father is present, the partner's role and resulting risks; (4) women's physical and mental state after childbirth and how it changes; (5) paternal depression; (6) how to prevent unintended injuries to the baby at home; (7) tips for combining childcare and work; and (8) the advantages of male engagement in childcare. The booklet for this study has been produced with reference to data from interviews previously carried out by the investigators with men who have experience of engaging in childcare, the Father and Child Health Handbook distributed by a number of Japanese local governments, and the results of studies of paternal depression in Japan and elsewhere, with additional scrutiny by researchers involved in maternal and child public health and midwives.

No intervention will be carried out for the participants in the control group. However, no restrictions will be placed on participation by members of both the intervention and control groups in the prenatal parenting classes routinely offered at the obstetric clinic.

\section{Outcome measurements}

\section{Primary outcome}

The primary outcome of this study is the proportion of new mothers' partners who are determined to be at risk of postnatal depression at either 1 or 3 months after childbirth. Postnatal depression will be measured by using the Edinburgh Postnatal Depression Scale (EPDS) ${ }^{26}$ Postpartum depression is defined as an EPDS score of $\geq 8$, based on a previous study that used the same criterion. ${ }^{27}$ According to this previous study, the sensitivity and specificity of this cut-off score for Japanese fathers were $81.8 \%$ and $94.1 \%$, respectively.

\section{Secondary outcomes}

Secondary outcomes include the risk of postnatal depression in the new mothers themselves, quality of life (QOL) of new mothers and their partners, partners' engagement in housework and childcare, bonding, tendency for child abuse and parenting stress on the part of new mothers. SPSS and STATA will be used for statistical analysis. QOL will be evaluated by WHOQOL26 (WHOQOL-BREF Japanese version), ${ }^{28}$ childcare participation by the Father's Child-Rearing Behavior Scale, ${ }^{29}$ bonding by the Japanese version of the Mother-to-Infant Bonding Scale (MIBS-J), ${ }^{30}$ parenting stress by the Parenting Stress Index Short Form (PSI-SF) ${ }^{31}$ and abuse by the Child Maltreatment Scale (CMS). ${ }^{32}$

WHOQOL-BREF in Japanese consists of four factors and its reliability has been evaluated among Japanese male and female $(0.66-0.75) .{ }^{33}$ We will assess childcare participation using one of two factors in the Father's child-rearing behaviour scale. The factor contains 15 items regarding childcare and housework and the internal reliability is $0.90 .^{29}$ MIBS-J is widely used at 
postnatal check-up in Japan and is composed of two factors. The internal reliabilities among Japanese fathers were 0.72 and 0.61 , respectively. ${ }^{34}$ PSI-SF comprises two factors including 19 items. The internal reliability $(0.82)$ and the concurrent validity were confirmed. ${ }^{35}$ CMS consists of 17 items and its reliability has been evaluated among Japanese women (0.63-0.77). ${ }^{36}{ }^{37}$ However, the reliability and validity of PSI-SF and CMS have not been verified among Japanese fathers.

\section{Sample size calculation}

A previous Japanese study of fathers in postpartum period found that the proportion of men judged to be suffering from postpartum depression in terms of their EPDS score 4 months after childbirth was $13.6 \%,{ }^{2}$ and a study carried out in the same geographical area as our study found that the period prevalence from a few days to 3 months after childbirth was $16.7 \% .^{3}$ We therefore assume that the period prevalence for postnatal depression (EPDS score $\geq 8$ ) among men at 1 and 3 months after childbirth in the absence of intervention (control group) will be $16.7 \%$. Calculated on the basis of a $5 \%$ significance level and $80 \%$ power, the number of participants (in the intervention group) required to demonstrate that the intervention reduced the rate of postnatal depression compared with the control group to $6 \%$ would be 148 men (148 couples), to $7 \%$ would be 189 men (189 couples), to $8 \%$ would be 241 men (241 couples), and to $9 \%$ would be 317 men (317 couples).

When estimating efficacy, no previous studies carried out in similar institutions are available. Studies that carried out interventions for partners at similar points during gestation varied widely in terms of the RCT intervention programme and its results, had either small sample sizes of around 50 participants in each group, carried out an intervention programme comprising parenting classes lasting several hours, or provided around 10 hours of standard education for members of the control group. Statistically significant effects of intervention for partners of pregnant women have been confirmed, such as a reduction of the adjusted OR for men with antenatal fear to $0.3,{ }^{38}$ and a $10 \%$ decrease in postnatal anxiety score among fathers. ${ }^{19}$

Given the lack of preceding studies providing sufficient evidence and taking feasibility into account, we assume that the rate of postnatal depression in this study would be decreased by around half $(8 \%)$. In this case, and assuming a dropout rate from follow-up surveys of $15 \%$, the required sample size for each group is 277 men. The overall target enrolment for the study is therefore 554 men ( 554 couples). Recruitment will be continued until the target sample size is achieved and the required number of participants has been secured.

\section{Handling of adverse events}

The intervention programme carried out in this study consists of the distribution of a booklet to participants with the objective of providing them with information.
On the basis of the stipulations of the Ethical Guidelines for Medical and Health Research Involving Human Subjects set out in 2014 by the Ministry of Education, Culture, Sports, Science and Technology and the Ministry of Health, Labour and Welfare, Japan, distribution of a booklet constitutes 'minor invasiveness'. It is therefore not expected that the intervention in this study will result in a clear risk to the life of the study participants. However, if the collected data are judged to reveal severe mental health problems or a tendency to abuse on the part of a participant, the survey results will immediately be provided to the participating institution as feedback, which will investigate whether or not the provision of care or support is required. The possibility to share the data depending on the results of assessment will be explained to the study participants when we conduct the recruitments.

\section{Data management}

All the information gathered from consent forms and questionnaires will be inputted into and managed on a computer that is not connected to the internet. To back up the input data, it will also be stored on a separate device. The electronic files containing these data will be password-protected and accessible only to authorised members of the research group. Range checks for data values will be carried out to improve data quality. The collected paper consent forms and questionnaires will be securely stored and will be destroyed by shredding at the end of the study.

\section{Statistical analysis}

Data analysis will be carried out using the intention-to-treat approach. Data for basic characteristics obtained from the baseline survey will be used to compare frequencies between the intervention and control groups. A $\chi^{2}$ test will be used to test for differences between the intervention and control groups in the risk of paternal depression (the primary outcome) and other category variables, and Student's t-test and the Mann-Whitney U-test will be used for continuous variables. When performing multivariate analysis to adjust for variables that have not been appropriately randomised, multiple logistic regressions will be used for category variables and multiple linear regressions for continuous variables. Subgroup analysis for primary and secondary outcomes will be performed by dividing the participants into primipara and multipara and investigating differences between the intervention and control groups. SPSS statistics V.19.0 (IBM SPSS Statistics for Windows, IBM Corp, Armonk, New York) will be used for all data analysis.

\section{Monitoring}

According to the Ethical Guidelines for Medical and Health Research Involving Human Subjects, ${ }^{39}$ the intervention programme in this study will correspond to 'minor invasiveness,' and the risk to participants will be 
kept to the minimum. None of the investigators taking part in this study have any conflict of interest to report. For these reasons, a monitoring committee has not been established for this study.

\section{DISSEMINATION}

\section{Protocol amendments}

The study protocol has been registered with the UMIN Clinical Trials Registry (UMIN-CTR), and should any amendments be made to the protocol, they will be recorded in the UMIN-CTR update history.

\section{Informed consent}

Written informed consent will be obtained from the pregnant women and their partners who form the participants of this study. Before consent is obtained, staff from the participating institution will give an oral explanation outlining the study to one or the other of the participant pairs and hand them an information sheet. Participants sign the consent form if they agree to cooperate with the study after having read the information sheet and understanding the outline and aims of the study, the fact that participation is voluntary, and that their privacy will be protected.

\section{Confidentiality}

Data obtained from consent forms and elsewhere that might identify individual participants, such as names and addresses, will be linkably anonymised using the study ID and managed separately from the data set used for purposes such as analysis. Data that might identify individuals will only be accessible by the staff of the participating institution and designated study staff. The linkably anonymised final trial data set will be accessible by all the contributors to this study. Researchers other than contributors may only access the final trial data set for the purpose of data analysis with the permission of the principal investigator.

\section{Dissemination}

The researchers will endeavour to publish the results of this study in statistical form that does not enable the identification of individuals, by presenting them at scientific conferences in Japan and overseas and submitting them as papers to international journals. The analytical results will also be provided as feedback to the participating institution and nearby administrative agencies, which will use them as resources for future healthcare and policies related to maternal and child public health.

Acknowledgements The authors thank Yoko Nakano, Koji Sekikawa and Mai Mizukoshi for their support in the preparation of the booklet used for the intervention programme. They are also grateful to the members of the Department of Health Policy at the National Center for Child Health and Development, Tokyo, Japan for their valuable comments during the preparation of the study protocol.
Contributors KT developed the intervention programmes in this study. KT and MS conducted the previous study regarding prevalence of paternal depression in Japan. KT, MO, NS, MS and HS participated in the design of this study. KT, MS and HS prepared all the questionnaires. KT, MO and NS developed the procedure of this study. KT and MS mainly wrote the first draft of the manuscript. All the authors reviewed and revised the draft, and gave final approval for publication of this protocol. All contributors will have the right to access the final data set of this study.

Funding This work was supported by JSPS KAKENHI grants (grant number 25862216).

Competing interests None declared.

Ethics approval This trial was approved in February 2016 by the Institutional Review Board and Ethics Committee of the National Center for Child Health and Development in Japan (approval number 1071).

Provenance and peer review Not commissioned; externally peer reviewed.

Data sharing statement This manuscript is a study protocol. We will correct data from now on.

Open Access This is an Open Access article distributed in accordance with the Creative Commons Attribution Non Commercial (CC BY-NC 4.0) license, which permits others to distribute, remix, adapt, build upon this work noncommercially, and license their derivative works on different terms, provided the original work is properly cited and the use is non-commercial. See: http:// creativecommons.org/licenses/by-nc/4.0/

\section{REFERENCES}

1. Paulson JF, Bazemore SD. Prenatal and postpartum depression in fathers and its association with maternal depression: a meta-analysis. JAMA 2010;303:1961-9.

2. Nishimura A, Fujita $Y$, Katsuta $M$, et al. Paternal postnatal depression in Japan: an investigation of correlated factors including relationship with a partner. BMC Pregnancy Childbirth 2015;15:128.

3. Suto M, Isogai E, Mizutani F, et al. Prevalence and Factors Associated With Postpartum Depression in Fathers: A Regional, Longitudinal Study in Japan. Res Nurs Health 2016;39:253-266.

4. Quevedo L, da Silva RA, Coelho F, et al. Risk of suicide and mixed episode in men in the postpartum period. $J$ Affect Disord 2011;132:243-6.

5. Nath S, Russell G, Ford T, et al. Postnatal paternal depressive symptoms associated with fathers' subsequent parenting: findings from the Millennium Cohort Study. $\mathrm{Br} J$ Psychiatry 2015;207:558-9.

6. Davis RN, Davis MM, Freed GL, et al. Fathers' depression related to positive and negative parenting behaviors with 1-year-old children. Pediatrics 2011;127:612-18.

7. Paulson JF, Bazemore SD, Goodman JH, et al. The course and interrelationship of maternal and paternal perinatal depression. Arch Womens Ment Health 2016.

8. Pinheiro RT, Magalhães PV, Horta BL, et al. Is paternal postpartum depression associated with maternal postpartum depression? Population-based study in Brazil. Acta Psychiatr Scand 2006;113:230-2.

9. Giallo R, D'Esposito F, Cooklin A, et al. Psychosocial risk factors associated with fathers' mental health in the postnatal period: results from a population-based study. Soc Psychiatry Psychiatr Epidemiol 2013;48:563-73.

10. Liu C, Cnattingius S, Bergström M, et al. Prenatal parental depression and preterm birth: a national cohort study. BJOG 2016.

11. Kvalevaag AL, Ramchandani PG, Hove O, et al. Paternal mental health and socioemotional and behavioral development in their children. Pediatrics 2013;131:e463-9.

12. Paulson JF, Keefe HA, Leiferman JA. Early parental depression and child language development. J Child Psychol Psychiatry 2009;50:254-62.

13. Ramchandani P, Psychogiou L. Paternal psychiatric disorders and children's psychosocial development. Lancet 2009;374:646-53.

14. van den Berg MP, van der Ende J, Crijnen AA, et al. Paternal depressive symptoms during pregnancy are related to excessive infant crying. Pediatrics 2009;124:e96-103.

15. Fujiwara T, Okuyama M, Takahashi K. Paternal involvement in childcare and unintentional injury of young children: a population-based cohort study in Japan. Int J Epidemiol 2010;39:588-97. 
16. Ito J, Fujiwara T, Barr RG. Is paternal infant care associated with breastfeeding? A population-based study in Japan. J Hum Lact 2013;29:491-9.

17. Dennis CL, Dowswell T. Psychosocial and psychological interventions for preventing postpartum depression. Cochrane Database Syst Rev 2013;(2):CD001134.

18. Aveyard $\mathrm{P}$, Lawrence $\mathrm{T}$, Evans $\mathrm{O}$, et al. The influence of in-pregnancy smoking cessation programmes on partner quitting and women's social support mobilization: a randomized controlled trial [ISRCTN89131885]. BMC Public Health 2005;5:80.

19. Li HT, Lin KC, Chang SC, et al. A birth education program for expectant fathers in Taiwan: effects on their anxiety. Birth 2009;36:289-96.

20. Wolfberg AJ, Michels KB, Shields W, et al. Dads as breastfeeding advocates: results from a randomized controlled trial of an educational intervention. Am J Obstet Gynecol 2004;191:708-12.

21. Bergström M, Kieler H, Waldenström U. Effects of natural childbirth preparation versus standard antenatal education on epidural rates, experience of childbirth and parental stress in mothers and fathers: a randomised controlled multicentre trial. BJOG 2009;116:1167-76.

22. Doherty WJ, Erickson MF, LaRossa R. An intervention to increase father involvement and skills with infants during the transition to parenthood. J Fam Psychol 2006;20:438-47.

23. Daley-McCoy C, Rogers M, Slade P. Enhancing relationship functioning during the transition to parenthood: a cluster-randomised controlled trial. Arch Womens Ment Health 2015;18:681-92.

24. Maycock B, Binns CW, Dhaliwal S, et al. Education and support for fathers improves breastfeeding rates: a randomized controlled trial. J Hum Lact 2013;29:484-90.

25. Tohotoa J, Maycock B, Hauck YL, et al. Can father inclusive practice reduce paternal postnatal anxiety? A repeated measures cohort study using the Hospital Anxiety and Depression Scale. BMC Pregnancy Childbirth 2012;12:75.

26. Cox JL, Holden JM, Sagovsky R. Detection of postnatal depression. Development of the 10-item Edinburgh Postnatal Depression Scale. Br J Psychiatry 1987;150:782-6.

27. Nishimura A, Ohashi K. Risk factors of paternal depression in the early postnatal period in Japan. Nurs Health Sci 2010;12:170-6.

28. Tazaki M, Nakane M. WHOQOL 26 Manual Revised. 1997 Tokyo: Kaneko Shobou Pub (in Japanese).
29. Naruse T, Arimoto A, Watai I, et al. Fathers' child-rearing behavior and influencing factors. Nihon Koshu Eisei Zasshi 2009;56:402-10. (in Japanese)

30. Yoshida K, Yamashita H, Conroy S, et al. A Japanese version of Mother-to-Infant Bonding Scale: factor structure, longitudinal changes and links with maternal mood during the early postnatal period in Japanese mothers. Arch Womens Ment Health 2012;15:343-52.

31. Abidin R. Parenting Stress Index: Professional Manual. 3rd edn. Odessa: Psychological Assessment Resources, 1995.

32. Tokunaga M, Ohara M, Kayama M, et al. Survey of child maltreatment among general population in Greater Tokyo. Kosei no Shihyo 2000;47:3-10. (in Japanese)

33. Skevington SM, Lotfy M, O'Connell KA. The World Health Organization's WHOQOL-BREF quality of life assessment: psychometric properties and results of the international field trial. A report from the WHOQOL group. Qual Life Res 2004;13:299-310.

34. Kitamura T, Takegata M, Haruna M, et al. The Mother-Infant Bonding Scale: factor structure and psychosocial correlates of parental bonding disorders in Japan. J Child Fam Stud 2015;24:393-401.

35. Araki A, Kanematsu Y, Yokosawa S, et al. A study for developing parenting stress-short form scale. J Child Health 2005;64:408-16. (in Japanese)

36. Fujiwara T, Kasahara M, Tsujii $\mathrm{H}$, et al. Association of maternal developmental disorder traits with child mistreatment: a prospective study in Japan. Child Abuse Negl 2014;38:1283-9.

37. Watanabe $Y$, Kayama M, Sagami A, et al. Child abuse and risk factors: a survey in Tokyo metropolitan area. Jpn Bull Soc Psychiatry 2002;10:239-46. (in Japanese)

38. Bergström M, Rudman A, Waldenström U, et al. Fear of childbirth in expectant fathers, subsequent childbirth experience and impact of antenatal education: subanalysis of results from a randomized controlled trial. Acta Obstet Gynecol Scand 2013;92:967-73.

39. Ministry of Education, Culture, Sports, Science and Technology/ Ministry of Health, Labour and Welfare. Ethical Guidelines for Medical and Health Research Involving Human Subjects. 2014 http://www.mhlw.go.jp/file/06-Seisakujouhou-10600000Daijinkanboukouseikagakuka/0000080278.pdf (accessed 24 May 2016). (in Japanese) 